\title{
Measuring or Monitoring of Novel Anticoagulants: Which Laboratory Test to Request?
}

\author{
Paul P. Dobesh $\cdot$ Kimberly J. Terry
}

Published online: 5 October 2013

(c) Springer Science+Business Media New York 2013

\begin{abstract}
Oral anticoagulants are now available with predictable pharmacokinetic and pharmacodynamic profiles, therefore eliminating the need for routine therapeutic monitoring. There are a number of clinical scenarios in which the ability to measure the pharmacodynamic and pharmacologic activity of these agent would be desirable. Since these new oral anticoagulants do not impact traditional coagulation assays like warfarin or heparins, and impact the assays differently among agents, measuring and monitoring creates a challenge for clinicians who care for these patients in the emergency or hospitalized setting. Understanding the effect of these agents, the available assays, and the interpretation of results is an element of their safe and effective use.
\end{abstract}

Keywords Anticoagulants - Blood coagulation tests . Direct thrombin inhibitors · Dabigatran · Rivaroxaban . Apixaban

\section{Introduction}

In recent years, anticoagulation management has broadened beyond the use of traditional oral vitamin $\mathrm{K}$ antagonists (VKA). New oral anticoagulants produce their effects

\section{P. P. Dobesh $(\square)$}

Department of Pharmacy Practice, College of Pharmacy, University of Nebraska Medical Center, 986045 Nebraska Medical Center, Omaha, NE 68198-6045, USA

e-mail: pdobesh@unmc.edu

\section{K. J. Terry}

Department of Pharmacy Services, Brigham and Women's

Hospital, 75 Francis St., Boston, MA 02115, USA

e-mail: kjterry@partners.org on specific factors in the coagulation cascade and have demonstrated clinical efficacy in the management of several thromboembolic conditions, including the prevention of venous thromboembolism (VTE) in patients undergoing hip or knee arthroplasty [1-6], stroke prevention in patients with nonvalvular atrial fibrillation (AF) [7-9], and the treatment of VTE [10-12]. Dabigatran, rivaroxaban, and apixaban are currently approved for use in the USA and many other countries, with edoxaban being the investigational agent farthest along in development (Table 1).

\section{New Oral Anticoagulants}

\section{Direct Thrombin Inhibitors}

Dabigatran etexilate is an oral prodrug that exerts its anticoagulant effect by directly inhibiting both free and clot-bound thrombin [13]. As a direct thrombin inhibitor (DTI) it inhibits coagulation by preventing fibrinogen to fibrin cleavage, activation of clotting factors, and thrombin-induced platelet aggregation. The prodrug is rapidly absorbed and converted to its active form, dabigatran, by plasma and hepatic esterases [14]. Dabigatran has an oral bioavailability of only $6-8 \%$ and reaches peak plasma concentrations in $1 \mathrm{~h}$ on an empty stomach or $2 \mathrm{~h}$ after meals. It does not rely on cytochrome P450 (CYP)mediated mechanisms for metabolism, but relies rather on hepatic glucuronidation [14]. Patients concomitantly taking P-glycoprotein inhibitors may be at risk of increased plasma concentrations and bleeding. Dabigatran is dependent on renal excretion for $80 \%$ of its elimination, with a serum half-life of $12-17 \mathrm{~h}$ [14]. In the phase II PETHRO study, dabigatran, $150 \mathrm{mg}$ twice daily, produced peak plasma concentrations of $184 \mathrm{ng} / \mathrm{mL}$ (range 
Table 1 Pharmacokinetic, pharmacodynamic, and clinical characteristics of new oral anticoagulants

\begin{tabular}{|c|c|c|c|c|}
\hline Drug & Dabigatran & Rivaroxaban & Apixaban & Edoxaban \\
\hline $\begin{array}{l}\text { Mechanism of } \\
\text { action }\end{array}$ & Direct thrombin inhibitor & Direct factor Xa inhibitor & Direct factor Xa inhibitor & Direct factor Xa inhibitor \\
\hline Bioavailability & $6-7 \%$ & $66 \%$ without food, $>80 \%$ with food & $50 \%$ & $62 \%$ \\
\hline$T_{\max }(\mathrm{h})$ & 1.5 & $2-3$ & $2-3$ & 1.2 \\
\hline$t_{1 / 2}(\mathrm{~h})$ & $12-14$ & $9-13$ & $8-15$ & $8-11$ \\
\hline $\begin{array}{l}\text { Protein binding } \\
(\%)\end{array}$ & 35 & 90 & 87 & 50 \\
\hline Metabolism & $\begin{array}{l}\text { P-glycoprotein substrate } \\
\text { glucuronidation }\end{array}$ & $\begin{array}{l}\text { CYP3A4/CYP3A5 } \\
\text { P-glycoprotein substrate }\end{array}$ & $\begin{array}{l}\text { CYP3A4/CYP3A5 } \\
\text { P-glycoprotein substrate }\end{array}$ & P-glycoprotein substrate \\
\hline Elimination & Renal $\sim 80 \%$ & $\begin{array}{l}\text { Renal } \sim 35 \% \\
\text { Hepatic } \sim 65 \%\end{array}$ & $\begin{array}{l}\text { Renal } \sim 25 \% \\
\text { Hepatic } \sim 75 \%\end{array}$ & $\begin{array}{l}\text { Renal } \sim 40 \% \\
\text { Hepatic } \sim 60 \%\end{array}$ \\
\hline $\begin{array}{l}\text { Preferred } \\
\text { laboratory tests } \\
\text { for } \\
\text { measurement }\end{array}$ & $\begin{array}{l}\text { aPTT } \\
\text { TT } \\
\text { ECT }\end{array}$ & $\begin{array}{l}\text { PT } \\
\text { Chromogenic anti-factor Xa assay }\end{array}$ & $\begin{array}{l}\text { Chromogenic anti- } \\
\text { factor Xa assay } \\
\text { dPT }\end{array}$ & $\begin{array}{l}\text { PT? } \\
\text { Chromogenic anti- } \\
\text { factor Xa assay? }\end{array}$ \\
\hline $\begin{array}{l}\text { Potential } \\
\text { laboratory tests } \\
\text { for monitoring }\end{array}$ & $\begin{array}{l}\text { Chromogenic } \\
\text { antithrombin assay with } \\
\text { calibrators and controls }\end{array}$ & $\begin{array}{l}\text { PT with calibrators and controls } \\
\text { Chromogenic anti-factor Xa assay } \\
\text { with calibrators and controls }\end{array}$ & $\begin{array}{l}\text { Chromogenic anti- } \\
\text { factor Xa assay with } \\
\text { calibrators and controls }\end{array}$ & $\begin{array}{l}\text { PT with calibrators and } \\
\text { controls? } \\
\text { Chromogenic anti- } \\
\text { factor Xa assay with } \\
\text { calibrators and controls? }\end{array}$ \\
\hline $\begin{array}{l}\text { Thromboembolic } \\
\text { conditions }\end{array}$ & Stroke prevention in $\mathrm{AF}$ & $\begin{array}{l}\text { Prevention of VTE in major } \\
\text { orthopedic surgery, stroke } \\
\text { prevention in AF, treatment of VTE }\end{array}$ & Stroke prevention in $\mathrm{AF}$ & Investigational \\
\hline
\end{tabular}

The activated partial thromboplastin time $(a P T T)$ loses sensitivity at higher dabigatran plasma concentrations. The thrombin time (TT) is oversensitive to moderate to high dabigatran plasma concentrations. The prothrombin time $(P T)$ loses sensitivity at lower rivaroxaban plasma concentrations and longer times after dosing. There is also variability depending on the thromboplastin used, which is not corrected by the international normalized ratio.

$A F$ atrial fibrillation, $C Y P$ cytochrome $\mathrm{P} 450, d P T$ diluted prothrombin time, $d T T$ diluted thrombin time, ECT ecarin clotting time, VTE venous thromboembolism

${ }^{a}$ Monitoring for the new oral anticoagulants is generally not needed, but may be desirable in certain clinical settings. At the time of writing, these tests are still for research purposes only.

b Although all of the agents in the table have been evaluated for prevention of VTE in major orthopedic surgery, stroke prevention in AF, and treatment of VTE, only the indications approved by the US Food and Drug Administration are listed here.

$64-443 \mathrm{ng} / \mathrm{mL}$ ) and trough concentrations of $90 \mathrm{ng} / \mathrm{mL}$ (range $31-225 \mathrm{ng} / \mathrm{mL}$ ) [15••]. Elderly subjects (mean age 68 years) receiving the same dose demonstrated peak plasma concentrations of $254 \pm 71 \mathrm{ng} / \mathrm{mL}$ for men and $285 \pm 81 \mathrm{ng} / \mathrm{mL}$ for women, and trough concentrations of approximately $80 \mathrm{ng} / \mathrm{mL}$ [14]. Although there are minor differences between dabigatran plasma levels achieved in different settings (healthy subjects, AF, VTE prevention), a study comparing exposure in these patient groups did not find statistically significant differences [16].

\section{Direct Factor Xa Inhibitors}

Rivaroxaban, apixaban, and edoxaban are classified as direct factor Xa (FXa) inhibitors (Table 1). By inhibiting FXa directly (not needing to first bind to antithrombin), agents of this class are able to inhibit both free and prothrombinase-bound FXa, and therefore inhibiting the conversion of prothrombin into thrombin [17]. Rivaroxaban is rapidly absorbed after administration and is more than $90 \%$ protein-bound. Rivaroxaban has differing bioavailability on concomitant administration with food, $65 \%$ on an empty stomach and $100 \%$ with food, and reaches peak plasma concentrations between 2 and $4 \mathrm{~h}$ [18]. Doses of rivaroxaban greater than $10 \mathrm{mg}$ should be administered with meals. Rivaroxaban undergoes metabolism via CYP3A4/CYP3A5, and concomitant use with P-glycoprotein and CYP3A4 inhibitors or inducers should be assessed prior to initiation. The ROCKET-AF trial allowed concomitant administration of weak to moderate CYP3A4 inhibitors and did not show increases in bleeding risks, even in those with moderate renal impairment (creatinine clearance $30-50 \mathrm{~mL} / \mathrm{min}$ ) [8]. Early rivaroxaban studies 
indicated pharmacokinetic (PK) and pharmacodynamic (PD) parameters were consistent and predictable among different patient populations based on age, gender, weight, and ethnicity. In patients with renal or hepatic impairment, alterations in PK and PD parameters were noted [17]. Patients with moderate hepatic impairment (Child Pugh B) saw significant increases in FXa inhibition, leading to prolonged exposure [18]. Early data in subjects receiving rivaroxaban at the dose used for VTE prevention $(10 \mathrm{mg}$ once daily) demonstrated peak plasma concentrations of $125 \mathrm{ng} / \mathrm{mL}$ (range 112-184 ng/mL) and trough concentrations of $9 \mathrm{ng} / \mathrm{mL}$ (range $1-38 \mathrm{ng} / \mathrm{mL}$ ) [19]. Subjects receiving rivaroxaban at the dose used in the prevention of stroke in AF and VTE treatment (20 mg once daily) demonstrated peak plasma concentrations of $173 \mathrm{ng} / \mathrm{mL}$ (range 111-294 ng/mL) and trough concentrations of approximately $24 \mathrm{ng} / \mathrm{mL}$ [19].

The oral bioavailability of apixaban is roughly $50 \%$,


21]. Approximately $25 \%$ of the drug is eliminated renally. Similarly to rivaroxaban, apixaban is metabolized by CYP3A4. Concomitant use with moderate to strong CYP3A4 or P-glycoprotein inhibitors or inducers may alter plasma concentrations of apixaban.

Edoxaban is an investigational oral direct FXa inhibitor completing phase III studies (Table 1) [22]. The bioavailability or edoxaban is approximately $60 \%$ [23]. It undergoes minimal CYP3A4 metabolism, but there is no data describing dosing reductions or precautions with strong CYP3A4 or P-glycoprotein inhibitors or inducers. Phase II trial data have demonstrated the drug is eliminated by renal and hepatic routes.

\section{Monitoring or Measuring}

The new oral anticoagulants provide significant advantages over those of VKA. They have fewer drug and food interactions, more standard dosing, and less pharmacogenomic influence on response. Population studies have also shown that these agents produce a more predicable PK and PD profile, reducing the need for routine therapeutic monitoring [24-26]. Although the new oral anticoagulants do not have defined therapeutic ranges similar to those of VKA, assessment of the effect of the drug or drug concentrations may be desired in certain clinical situations. Measurement or monitoring of new oral anticoagulants may be warranted if patients present with a new thrombotic or hemorrhagic event, when patient adherence is to be checked, in overdose situations, in patients with reduced clearance due to renal or hepatic insufficiency, in patients requiring emergent and/or high-risk surgical procedures where bleeding could be catastrophic (neurosurgery), or in patients taking interacting drugs. An additional scenario very pertinent to the emergency department physician is the patient presenting with life-threatening hemorrhage. Owing to the high cost and high risk of administering potential reversal agents, it would be critical to know if the new oral anticoagulant was still providing a significant anticoagulant effect.

It is important to differentiate between the desire to provide monitoring of these new oral anticoagulants and the need to measure the effect of the drug at a given time. Monitoring implies laboratory testing to assess the drug's effect and to adjust the dosage to maintain anticoagulation within a therapeutic interval. This is typically not thought to be necessary with the new oral anticoagulants. Although it has yet to be evaluated, monitoring may be valuable in the future in patients with renal or liver insufficiency, in the very elderly, in patients taking interacting drugs, or possibly in patients needing these agents in addition to dual antiplatelet therapy. Measurement implies laboratory evaluation of the drug's effect, not necessarily to use the results to adjust the dosage, but simply to determine whether patients are underanticoagulated or overanticoagulated. This could apply to all of the clinical situations previously listed for the new oral anticoagulants. Assay techniques for measuring anticoagulants include clot-based assays, chromogenic assays, enzyme-linked immunosorbent assays, and high-performance liquid chromatography. The concentrations of new oral anticoagulants can be accurately determined by high-performance liquid chromatography methods [27]. However, these techniques are unsuitable for clinical routine use. Clot-based assays and chromogenic assays are most commonly used for clinical laboratory assessment.

\section{Clot-Based Coagulation Assays}

\section{Prothrombin Time}

The prothrombin time (PT) is a global assessment of the function of the extrinsic clotting cascade. The PT is a measure of the time in seconds for plasma to clot after addition of calcium and thromboplastin [28]. Thromboplastin contains tissue factor and phospholipids, which are needed to create FXa by factor VIIa. Thromboplastin reagents have differing sensitivities to coagulation factor deficiencies or inhibition owing to the different sources and methods for the preparation of the tissue factor [29]. These differences produce variable results for the PT for the same blood sample depending on the thromboplastin used. The international normalized ratio (INR) was developed to provide standardization of PT results when using VKA by providing an international sensitivity index (ISI) for each 
thromboplastin developed [28]. It is desirable to be able to use the PT for assessment of the new oral anticoagulants since the test is readily available in practically all clinical laboratories at any time of day and limited expertise is required to perform the test.

Dabigatran produces a concentration-dependent increase in the PT $[14,15 \bullet, 23-32]$. The dabigatran plasma concentration needed to double the PT is typically more than $600 \mathrm{ng} / \mathrm{mL}$. This concentration is significantly higher than what is typically seen with peaks in the treatment of AF. Dabigatran, $150 \mathrm{mg}$ twice daily, produces peak levels of approximately $200 \mathrm{ng} / \mathrm{mL}$ and generates a PT of 1.2 times the baseline value [33*•]. Although the PT can be prolonged with dabigatran, because of the low sensitivity of the test, there is very little prolongation at typically achieved plasma concentrations. Therefore, the PT is not appropriate for assessing the effects of dabigatran.

Since direct FXa inhibitors inhibit free FXa and FXa bound in the prothrombinase complex, it would be expected that the PT would be prolonged with agents of this class. The impact of rivaroxaban on the PT has been evaluated in a number of studies [34, 35, 36•]. Although rivaroxaban produces a concentration-dependent prolongation of the PT in a liner fashion, the slope of the concentration-effect relationship varies considerably depending on the thromboplastin used [26, 34, 35]. The reason for this variability is based on the different ISI for each of the thromboplastins evaluated, and therefore, the different sensitivities to the anticoagulant effect of the direct FXa inhibitors. Unfortunately, this variability is not corrected for by conversion of the PT to an INR as it is with VKA. In fact, multiple studies have demonstrated an increase in variability when the INR is applied to direct FXa inhibitors because of differential assay reagent sensitivities to rivaroxaban $[35,36 \cdot$, 37]. The most responsive thromboplastins seem to be Neoplastin CI Plus ${ }^{\circledR}$ (Diagnostica Stago, Asniéres-sur-Seine, France) and RecombiPlasTin ${ }^{\circledR}$ (Instrumentation Laboratory, Bedford, MA, USA), whereas the least responsive consistently is Inno$\operatorname{vin}^{\circledR}$ (Siemens Healthcare Diagnostics, Deerfield, IL, USA) $[34,38]$.

Although variability in the PT response to rivaroxaban depending on the thromboplastin used is one limitation to the test, there are others. The PT does not seem to be sensitive to lower concentrations of rivaroxaban, and the effect of rivaroxaban on the test is short-lived [39, 40]. In human plasma spiked with rivaroxaban, the effect on the PT can be observed at rivaroxaban plasma concentrations between 50 and $700 \mathrm{ng} / \mathrm{mL}$. The test lacks accuracy and precision at lower (trough) rivaroxaban plasma concentrations [41]. A study with patients receiving rivaroxaban, $10 \mathrm{mg}$ once daily, demonstrated a prolongation of the PT $2 \mathrm{~h}$ after study medication (peak), but the PT was no longer prolonged $12 \mathrm{~h}$ later [40]. After a $30 \mathrm{mg}$ dose of rivaroxaban, the PT at $12 \mathrm{~h}$ was only 1.2-fold higher compared with the baseline, and returned to the baseline by $24 \mathrm{~h}$ [42•]. Therefore, it cannot be assumed that patients without an elevated PT are not taking their rivaroxaban as prescribed or that there are "low" levels of the drug in the patient. The PT assay may be useful for performing qualitative measurements of maximum plasma drug concentrations (overdose), but is not recommended for determining minimum plasma concentrations (safety for surgery).

In an attempt to standardize PT results, a calculation of the ISI for commercial thromboplastins specific for rivaroxaban has been evaluated [38, 43•]. This ISI is used to convert the PT ratio into a standardized scale similar to that used for VKA. Even if an INR value for the direct FXa inhibitors is developed to standardize the PT results, there is no reason to believe that the "therapeutic range" of 2.0-3.0 will apply to these agents. Developing an INR for direct FXa inhibitors would only be the first step to determining cutoffs for efficacy and safety from new clinical trials. It is also possible the different direct FXa inhibitors will produce different cutoffs for these outcomes.

Another attempt to standardize the PT test results, and even develop potential monitoring of direct FXa inhibitors, is to modify the PT with the use of specific calibrators. This allows the results of PT measurements to be expressed as plasma concentrations of rivaroxaban in nanograms per milliliter [44, 45]. Calibration plasmas obtained by spiking pooled normal plasma with known and increasing amounts of rivaroxaban are commercially available in lyophilized form. These calibrators would help translate clotting times obtained with any given method/reagent into concentrations of the drug by means of local calibration curves. This would have the benefit of making possible a simple and widely available test to assess the PKs and PDs of agents of this class [34]. By use of these calibrators and controls, the PT would go from providing simply qualitative data to providing more quantitative data for making patient care decisions.

Not all of the direct FXa inhibitors seem to impact the PT to the same extent. Whereas edoxaban appears to have the same impact on the PT as rivaroxaban [23], darexaban has a more pronounced impact on the PT [36•]. Apixaban also produces a prolongation of the PT, but the test is less sensitive to apixaban. The concentration of apixaban needed to create a doubling of the PT is almost twice that of rivaroxaban, even with more sensitive thromboplastins such as Neoplastine CI and Neoplastine CI Plus [46]. Therefore, there is almost no meaningful increase in the PT unless supratherapeutic plasma concentrations of apixaban are achieved [46, 47]. The thromboplastin sensitivities of various direct FXa inhibitors differ considerably [48]. 
Therefore, comparisons between drugs in this class are not possible. Apixaban has been evaluated with the use of a diluted PT (dPT) test, also known as a modified PT test, which uses a lower concentration of thromboplastin regent than that used in the PT test in order to increase the sensitivity [47]. It may be worth noting that the conditions of the dPT test might be closer to physiological conditions than those of the standard PT test [34]. Although apixaban had little impact on the PT, there was a concentrationdependent impact on the $\mathrm{dPT}$ in healthy volunteers. A dose of $50 \mathrm{mg}$ apixaban created a 2.6 -fold increase in the dPT, and was found to be more sensitive for apixaban than the PT test [47].

\section{Activated Partial Thromboplastin Time}

The activated partial thromboplastin time (aPTT) is a global measure of the function of the intrinsic clotting cascade. The test is performed by adding a surface activator (Celite, kaolin, ellagic acid, or silica) and a partial thromboplastin to citrated plasma. The thromboplastin is called "partial" because it contains phospholipid, but unlike the thromboplastin used for the PT, it does not include tissue factor. There is an incubation period to allow activation of factor XI, factor XII, prekallikrein, and high molecular weight kininogen. Calcium is then added to the sample, and the clotting time is measured [28].

A number of studies have demonstrated that dabigatran prolongs the aPTT in a curvilinear fashion $[14,15 \bullet$, 23-32]. There is a more linear response of the aPTT at lower dabigatran plasma concentrations, but then there is a leveling off at concentrations typically above $200 \mathrm{ng} / \mathrm{mL}$ (depending on the partial thromboplastin used) [33・•]. The responsiveness of the test is adequate because dabigatran plasma concentrations of $200 \mathrm{ng} / \mathrm{mL}$ prolong the aPTT by about 2.0-2.5 times the baseline value [33••]. In another study, the concentration needed to double the aPTT was calculated to be $227-286 \mathrm{ng} / \mathrm{mL}$ [30]. Owing to the variable sensitivity of the aPTT reagents, the corresponding dabigatran plasma levels of more than $67 \mathrm{ng} / \mathrm{mL}$ and more than $200 \mathrm{ng} / \mathrm{mL}$ may correlate with widely differing aPTT ranges (35-50 s for prophylaxis and 50-70 s $12 \mathrm{~h}$ after therapeutic administration of dabigatran twice daily) [14, $32,33 \cdot 0$. The flattening of the aPTT curve caused by lower sensitivity at higher dabigatran plasma concentrations makes it difficult to determine if overdose or accumulation of the drug has occurred. This flattening or curvilinear response to dabigatran has also been demonstrated with other DTI such as lepirudin and argatroban [49]. Patients with substantially elevated dabigatran levels (above $400 \mathrm{ng} / \mathrm{mL}$ ) almost always have marked prolongations of their aPTT values. Thus, the aPTT may not be suitable for accurately quantifying the anticoagulant effect of dabigatran, but may provide a qualitative assessment of the anticoagulant activity [15••].

Rivaroxaban has a weaker effect on the aPTT assay than on the PT assay $[26,34,35]$. Any impact on the aPTT also seems to be short-lived. In a study of healthy subjects, a dose of $80 \mathrm{mg}$ rivaroxaban (four times the dose used in practice) only produced a 1.5 -fold increase in the aPTT from the baseline [19]. The concentration of rivaroxaban needed to double the aPTT was three times than that needed to double the PT $(0.69 \mu \mathrm{M}$ vs $0.23 \mu \mathrm{M})$, demonstrating the higher sensitivity of the PT [17]. The aPTT also demonstrates weak sensitivity to measuring the effect of apixaban. When $50 \mathrm{mg}$ apixaban (ten times the dose used in practice) was given to healthy subjects, there was only a 1.2-fold increase in the aPTT from the baseline [48]. Of the direct FXa inhibitors evaluated, edoxaban probably has the largest impact on the aPTT. A dose of $60 \mathrm{mg}$, which may end up being used in practice, produced a 1.5 -fold increase in the aPTT $[22,23]$. On the basis of the lack of sensitivity of the aPTT to measure most direct FXa inhibitors, and the availability of better methods, the aPTT cannot be recommended for use in measuring agents of this class.

\section{Thrombin Time}

The thrombin time (TT), or thrombin clotting time, is evaluated by simply adding excess thrombin to the plasma sample and measuring the time for clot formation [28]. This test is readily available in most laboratories and is not technologically challenging to perform. Since the TT is a direct measure of thrombin activity, DTI such as dabigatran produce a pronounced prolongation of the TT. Owing to the high sensitivity of the test to dabigatran, the TT is good for detecting low to moderate plasma concentrations [15••]. This would make the TT test a useful test in qualitative assessments of dabigatran in situations such as needing to perform highly invasive surgery or providing fibrinolytic therapy for treatment of acute ischemic stroke. The TT is too sensitive to provide any assessment of high dabigatran plasma concentrations, as readings often exceed the maximum measurement time during peak concentrations of dabigatran $[15 \cdot 0]$. A dabigatran plasma concentration of $200 \mathrm{ng} / \mathrm{mL}$ may prolong the TT by nearly 15 -fold [33••].

Owing to the extensive sensitivity of the TT to DTI, a modified test called the diluted TT (dTT) is also used [50]. The dTT assay can be used to accurately measure dabigatran concentrations. Because of the linear dose-response curves over a wide concentration range, it can be used to accurately monitor both trough and peak dabigatran levels [31, 32, 33••, 51-53]. The Hemoclot ${ }^{\circledR}$ assay is a modified TT assay in which the plasma sample is diluted 1:8. Dilution of the test sample avoids the exaggerated sensitivity of assay response 
associated with the conventional TT test [53]. On the basis of these modifications, a dabigatran plasma concentration of $200 \mathrm{ng} / \mathrm{mL}$ produces about a threefold increase in the dTT from the baseline $[14,53]$. With the Hemoclot ${ }^{\circledR}$ assay, the calibration curve was shown to be linear up to the maximum concentration tested of $1,886 \mathrm{ng} / \mathrm{mL}$, which is approximately four times the concentration expected with the use of dabigatran in the treatment of VTE or stroke prevention in AF [53]. Commercial availability of dabigatran calibration standards and quality control samples makes possible the measurement of dabigatran concentrations rather than determining the clotting time response only, which may differ between laboratories. They also render the assay independent of specific coagulometers and eliminate between-laboratory variability $[51,53]$. Although external calibration of the Hemoclot ${ }^{\circledR}$ assay with hirudin makes possible determination of dabigatran concentrations expressed as "hirudin equivalents," direct calibration with stable, lyophilized dabigatran standards is more precise [14]. Because a direct FXa inhibitor would have no impact on excess thrombin introduced into the sample, these agents have no impact on the TT or dTT [40].

\section{Ecarin Clotting Time}

During the normal coagulation process, prothrombin is converted to thrombin in a two-step proteolytic process on the prothrombinase complex. The prothrombinase complex consists of $\mathrm{FXa}$, factor $\mathrm{Va}$, and calcium ions on a phospholipid membrane, typically found on platelets. The cleavage of prothrombin first at Arg-217 produces prothrombin 2, which is about half the size of prothrombin, and fragment 1.2 [54]. The prothrombin 2 intermediate is then cleaved at Arg-320 to create a stereochemical change and the active thrombin molecule. When the ecarin clotting time (ECT) assay is performed, ecarin is added to the sample to promote cleavage of free prothrombin first at Arg-320, therefore creating a meizothrombin intermediate that is not attached to the prothrombinase complex [54]. Ecarin is a purified metalloprotease from the venom of the viper Echis carinatus. Since meizothrombin has the same molecular weight as prothrombin, it is not inhibited by the large complex formed with antithrombin and unfractionated heparin (UFH), low molecular weight heparin (LWMH), or fondaparinux. Owing to the ability of a DTI to inhibit meizothrombin, the meizothrombin generated is neutralized in relation to the amount or concentration of DTI in the system [28, 54]. Even though many laboratories do not typically run this test and there is no FDA-approved ECT assay, ecarin is commercially available and the test can be run on most coagulometers.

Dabigatran and other DTI produce a linear increase in the ECT with increasing plasma concentrations [15••, 49].
The ECT is prolonged about threefold compared with the baseline at a dabigatran plasma concentration of $200 \mathrm{ng} /$ $\mathrm{mL}[15 \bullet \bullet, 33 \bullet \cdot$. A chromogenic variant of the ECT assay in which ecarin is added to a plasma sample and meizothrombin generation is measured with a chromogenic substrate has also been developed [55]. The test currently lacks standardization across different laboratories. The ECT and the chromogenic ecarin assay can be calibrated to dabigatran concentrations, and would be a suitable test for evaluating the anticoagulant activity of dabigatran [14]. Agents that inhibit FXa would not be expected to have any impact on the ECT.

\section{Chromogenic Assays}

Chromogenic anti-FIIa and anti-FXa assays have been developed, with the anti-FXa assay being used to measure plasma concentrations of UFH and LMWH. The anti-FXa assay uses a FXa substrate that has a chromophore connected to it. When FXa cleaves the chromogenic substrate, it releases the chromophore, producing a yellow color that can be detected with a spectrophotometer. The intensity of the yellow color produced is indirectly proportional to the amount of $\mathrm{FXa}$ present; therefore, the greater the amount of FXa inhibited, the lower the intensity of the color produced [55].

Since FXa is the target for direct FXa inhibitors, the anti-FXa assay should be an optimal test for agents of this class. Rivaroxaban (up to concentrations of $500 \mathrm{ng} / \mathrm{mL}$ ) produces a concentration-dependent effect in commercially available assays, which use bovine FXa, but sensitivity differs between assays $[34,37,56]$. The use of human FXa, instead of bovine $\mathrm{FXa}$, and other modifications produce a linear response to rivaroxaban up to $1,000 \mathrm{ng} / \mathrm{mL}$ [34]. LMWH calibration curves should not be used to interpolate assay results. Instead, the anti-FXa activity curve should be constructed using samples spiked with various known concentrations of the direct FXa inhibitor [34, 37].

Recent studies suggest that commercially available chromogenic FXa assays may be useful for the quantitative determination of rivaroxaban provided that a standard curve is generated with known amounts of rivaroxaban using rivaroxaban-spiked plasma samples as a calibrator. Controls for both low and high levels of rivaroxaban are also necessary, and the plasma concentration of rivaroxaban is expressed in nanograms per milliliter [41, 57, 58•]. With use of rivaroxaban calibrators and controls, rivaroxaban plasma concentrations can be reliably evaluated for concentrations ranging from 20 to $660 \mathrm{ng} / \mathrm{mL} \mathrm{[58 \bullet ].}$

A similar finding has been demonstrated for apixaban, showing that different anti-FXa assays from various manufacturers were consistent in their rank order when tested with different FXa inhibitors, suggesting that chromogenic 
anti-FXa assays are preferable to the PT assay for assessing apixaban exposure [37].

The anti-FIIa test is based on the measurement of residual thrombin by a synthetic chromogenic peptide on addition of excess thrombin to a patient's plasma sample. Chromogenic assays to measure dabigatran are currently in development, and a chromogenic assay to measure thrombin activity has been assessed for measuring the concentration of the DTI lepirudin and argatroban [49]. In this assay, samples are incubated with a thrombin substrate, and human thrombin is then added. Color development is measured, giving an inverse relationship with the concentration of thrombin inhibitor. Recent studies suggest that commercially available chromogenic thrombin assays may be useful for the quantitative determination of dabigatran activity, provided that a standard curve is generated with known dabigatran concentrations [32, 59].

\section{Conclusions}

Although the lack of a need for routine monitoring with the new oral anticoagulants is clearly an advantage to patients in the management of chronic disease, there are scenarios in emergency and hospital settings where being able to measure the PD effect of these agents is desired. For dabigatran, the aPTT can be used as a qualitative measurement, but the test lacks sensitivity at higher plasma concentrations. The opposite is true for the TT, in which the test is too sensitive at high concentrations. Therefore, in settings when the desire is to know if there is too much drug, such as in an overdose, when there is bleeding, or when deciding to administer a reversal agent, the aPTT will tell you there is drug in the patient (just not how much). If the desire is to know if the drug has cleared from the patient, a lack of prolongation of the TT would be helpful in assessing adherence in the case of a recurrent thrombotic event, the possibility of administering a fibrinolytic to a patient presenting with stroke, or safety to proceed to surgery. The ECT would also be an appropriate test, but is not available in many laboratories. If monitoring drug therapy is desired, such as in the setting of altered clearance or drug interactions, the dTT, or the chromogenic anti-FIIa assay in the future, would be the best assay. Using the PT or INR is not helpful in the setting of dabigatran.

For rivaroxaban, the PT can be used as a qualitative assessment, but the sensitivity of the test is poor at lower concentrations and beyond $12 \mathrm{~h}$ after dosing. There is also significant variation across thromboplastins. Therefore, the PT would be useful in the setting of an overdose, major bleeding, or deciding to administer a reversal agent, but lack of prolongation would not necessarily suggest safety for surgery or administering a fibrinolytic in the setting of stroke. The INR should not be used at this time, as it actually worsens the variability with the PT (opposite of warfarin). Currently the chromogenic anti-FXa assay would be the best test, but the standardization curve needs to be constructed for each FXa inhibitor, and the curves used for UFH or LMWH are not accurate. For apixaban, it appears that only the anti-FXa assay should be used as the PT lacks any sensitivity with this agent. There will be potential for monitoring direct $\mathrm{FXa}$ inhibitors with the use of the PT or chromogenic anti-FXa assays if appropriate calibrators and controls are used to standardize the tests. Widespread laboratory uptake of these calibrators and controls may take time.

\section{Compliance with Ethics Guidelines}

Conflict of Interest Paul P. Dobesh has received compensation from Janssen Pharmaceuticals, Boehringer Ingelheim, BMS/Pfizer, and Daiichi Sankyo for service as a consultant, and has received financial support via grants from Daiichi Sankyo and the Eli Lilly Alliance. Kimberly J. Terry declares that she has no conflict of interest.

Human and Animal Rights and Informed Consent This article does not contain any studies with human or animal subjects performed by any of the authors.

\section{References}

Papers of particular interest, published recently, have been highlighted as:

- Of importance

- Of major importance

1. Eriksson BI, Dahl OE, Rosencher N, RE-NOVATE Study Group, et al. Dabigatran etexilate versus enoxaparin for prevention of venous thromboembolism after total hip replacement: a randomised, double-blind, non-inferiority trial. Lancet. 2007;370:949-56.

2. The RE-MOBILIZE Writing Committee. Oral thrombin inhibitor dabigatran etexilate vs. North American enoxaparin regimen for prevention of venous thromboembolism after knee arthroplasty surgery. J Arthroplasty. 2009;24:1-9.

3. Eriksson BI, Borris LC, Friedman RJ, RECORD 1 Study Group, et al. Rivaroxaban versus enoxaparin for thromboprophylaxis after hip arthroplasty. N Engl J Med. 2008;358:75.

4. Turpie AAG, Lassen MR, Davidson BL, et al. Rivaroxaban versus enoxaparin for thromboprophylaxis after total knee arthroplasty (RECORD 4): a randomised trial. Lancet. 2009;373:1673-80.

5. Lassen MR, Gallus A, Raskob GE, et al. Apixaban versus enoxaparin for thromboprophylaxis after hip replacement. N Engl J Med. 2010;363:2487-98.

6. Lassen MR, Roskob GE, Gallus A, et al. Apixaban or enoxaparin for thromboprophylaxis after knee replacement. N Engl J Med. 2009;361:594-604.

7. Connolly SJ, Ezekowitz MD, Yusuf S, et al. Dabigatran versus warfarin in patients with atrial fibrillation. $\mathrm{N}$ Engl $\mathrm{J}$ Med. 2009;361:1139-51. 
8. Patel MR, Mahaffey KW, Garg J, et al. Rivaroxaban versus warfarin in nonvalvular atrial fibrillation. $\mathrm{N}$ Engl $\mathrm{J}$ Med. 2011;365:883-91.

9. Granger CB, Alexander JH, McMurray JJV, et al. Apixaban versus warfarin in patients with atrial fibrillation. $\mathrm{N}$ Engl $\mathrm{J}$ Med. 2011;365:981-92.

10. Schulman S, Kearon C, Kakkar AK, et al. Dabigatran versus warfarin in the treatment of acute venous thromboembolism. N Engl J Med. 2009;361:2342-52.

11. The EINSTEIN Investigators. Oral rivaroxaban for symptomatic venous thromboembolism. N Engl J Med. 2010;363:2499-510.

12. Agnelli C, Buller HR, Cohen A, et al. Oral apixaban for the treatment of acute venous thromboembolism. N Engl J Med. 2013;369(9):799-808.

13. Wienen W, Stassen JM, Priepke H, et al. In vitro profile and ex vivo anticoagulant activity of the direct thrombin inhibitor dabigatran and its orally active prodrug, dabigatran etexilate. Thromb Haemost. 2007;98:155-62.

14. Stangier J. Clinical pharmacokinetics and pharmacodynamics of the oral direct thrombin inhibitor dabigatran etexilate. Clin Pharmacokinet. 2008;47:285-95.

15. • van Ryn J, Stangier J, Haertter S, et al. Dabigatran etexilate-a novel, reversible, oral direct thrombin inhibitor: interpretation of coagulation assays and reversal of anticoagulant activity. Thromb Haemost. 2010;103:1116-27. This study is one of the most extensive for evaluating the impact of dabigatran on different commonly used coagulation assays. There are a number of good figures and tables that are used to summarize the data. There is also a good discussion on the connection to certain findings to typical therapeutic plasma concentrations that would be expected from doses of dabigatran used in practice. If someone wanted to read one study on the assessment of dabigatran with coagulation assays, this would be that paper.

16. Dansirkul C, Lehr T, Liesenfeld K-H, et al. A combined pharmacometric analysis of dabigatran etexilate in healthy volunteers and patients with atrial fibrillation or undergoing orthopaedic surgery. Thromb Haemost. 2012;107:775-85.

17. Perzborn E, Strassburger J, Wilmen A, et al. In vitro and in vivo studies of the novel antithrombotic agent BAY 59-7939-oral, direct factor Xa inhibitor. J Thromb Haemost. 2005;3:514-21.

18. Perzborn E, Roehrig S, Straub A, et al. The discovery and development of rivaroxaban, an oral, direct factor Xa inhibitor. Nat Rev Drug Discov. 2011;10:61-75.

19. Kubitza D, Becka M, Voith B, et al. Safety, pharmacodynamics, and pharmacokinetics of single doses of BAY 59-7939, an oral, direct factor Xa inhibitor. Clin Pharmacol Ther. 2005;78:412-21.

20. Raghavan N, Frost CE, Yu Z, et al. Apixaban metabolism and pharmacokinetics after oral administration to humans. Drug Metab Dispos. 2009;37:74-81.

21. Frost C, Nepal S, Wang J, et al. Safety, pharmacokinetics and pharmacodynamics of multiple oral dose of apixaban, a factor $\mathrm{Xa}$ inhibitor, in healthy subjects. Br J Clin Pharmacol 2013. doi:10. 1111/bcp.12106.

22. Ogata K, Mendell-Harary J, Tachibana M, et al. Clinical safety, tolerability, pharmacokinetics and pharmacodynamics of the novel factor Xa inhibitor edoxaban in healthy volunteers. J Clin Pharmacol. 2010;50:743-53.

23. Liesenfeld K-H, Schäfer HG, Trocóniz IF, et al. Effects of the direct thrombin inhibitor dabigatran on ex vivo coagulation time in orthopaedic surgery patients: a population model analysis. Br J Clin Pharmacol. 2006;62:527-37.

24. Mueck W, Becka M, Kubitza D, et al. Population model of the pharmacokinetics and pharmacodynamics of rivaroxaban-an oral, direct factor Xa inhibitor-in healthy subjects. Int J Clin Pharmacol Ther. 2007;45:335-44.
25. Mueck W, Borris LC, Dahl O, et al. Population pharmacokinetics and pharmacodynamics of once- and twice-daily rivaroxaban for the prevention of venous thromboembolism in patients undergoing total hip replacement. Thromb Haemost. 2008;100:453-61.

26. Helin T, Pakkanen A, Lassila R, Joutsi-Korhonen L. Laboratory assessment of novel oral anticoagulants: method suitable and variability between coagulation laboratories. Clin Chem. 2013;59:807-14.

27. Bates SM, Weitz JI. Coagulation assays. Circulation 2005;112: e53-e60.

28. Kamal AH, Tefferi A, Pruthi RK. How to interpret and pursue an abnormal prothrombin time, activated partial thromboplastin time, and bleeding time in adults. Mayo Clin Proc. 2007;82:864-73.

29. Lindahl TL, Baghael F, Fagerberg Blixter I, et al. Effects of the oral, direct thrombin inhibitor dabigatran on five common coagulation assays. Thromb Haemost 2011;105:371-8.

30. Green L, Lawrie AS, Patel R, et al. The effect of total hip/knee replacement surgery and prophylactic dabigatran on thrombin generation and coagulation parameters. Thromb Res. 2012;130: $775-9$.

31. Douxfils J, Mullier F, Robert S, et al. Impact of dabigatran on a large panel of routine or specific coagulation assays. Laboratory recommendations for monitoring of dabigatran etexilate. Thromb Haemost. 2012;107:985-97.

32. Stangier J, Rathgen K, Staehle H, et al. The pharmacokinetics, pharmacodynamics and tolerability of dabigatran etexilate, a new oral direct thrombin inhibitor, in healthy male subjects. Br J Clin Pharmacol. 2007;64:292-303.

33. •- Samama MM, Martinol J-L, LeFlem L, et al. Assessment of laboratory assays to measure rivaroxaban - an oral, direct factor Xa inhibitor. Thromb Haemost. 2010;103:815-25. This study is one of the most extensive for evaluating the impact of rivaroxaban on different commonly used coagulation assays. There are a number of good figures and tables that are used to summarize the data. If someone wanted to read one study on the assessment of dabigatran with coagulation assays, this would be that paper.

34. Hillarp A, Baghael F, Fagerberg Blixter I, et al. Effects of the oral, direct factor Xa inhibitor rivaroxaban on commonly used coagulation assays. J Thromb Haemost. 2011;9:133-9.

35. Harenberg J, Marx S, Weiss C, et al. Report of the Subcommittee of Control of Anticoagulation on the determination of the anticoagulant effects of rivaroxaban. J Thromb Haemost. 2012;10: 1433-6.

36. - Barrett YC, Wang Z, Frost C, Shenker A. Clinical laboratory measurement of direct factor $\mathrm{Xa}$ inhibitors: anti-Xa assay is preferable to prothrombin time assay. Thromb Haemost 2010;104:1263-71. This study evaluated commercial PT/INR and anti-FXa activity assays in patients with VTE. Apixaban plasma concentration was more strongly correlated with anti-FXa activity compared with the PT/INR.

37. Tripodi A, Chantarangkul V, Guinet C, Samama M. The international normalized ratio calibrated for rivaroxaban has the potential to normalize prothrombin time results for rivaroxabantreated patients: results of an in vitro study. J Thromb Haemost. 2011;9:226-8.

38. Eriksson BI, Quinlan DJ, Weitz JI. Comparative pharmacodynamics and pharmacokinetics of oral direct thrombin and facto $\mathrm{Xa}$ inhibitors in development. Clin Pharmacokinet. 2009;48:1-22.

39. Mani H, Hesse C, Stratmann G, Lindhoff-Last E. Rivaroxaban differentially influences ex vivo global coagulation assays based on administration time. Thromb Haemost. 2011;106:156-64.

40. Lindhoff-Last E, Samama MM, Ortel TL, et al. Assays for measuring rivaroxaban: their suitability and limitations. Ther Drug Monit. 2010;32:673-9. 
41. Graff J, von Hentig N, Misselwitz F, et al. Effects of the oral direct factor $\mathrm{Xa}$ inhibitor rivaroxaban on platelet-induced thrombin generation and prothrombinase activity. J Clin Pharmacol. 2007;47:1398-407.

42. - Harenberg J, Marx S, Krämer R, et al. Determination of an international sensitivity index of thromboplastin reagents using a WHO thromboplastin as calibrator for plasma spiked with rivaroxaban. Blood Coagul Fibrinolysis. 2011;22:637-41. This study is important because it demonstrates the different impact that the thromboplastins have on direct FXa inhibitors have on PT and INR compared to warfarin. The figures in this study are very informative and really get across the point that the same coagulation assay provides very different results between different classes of agents. The study is also interesting in that it exposes the reader to a potential future of using this approach in the care of patients receiving direct $F X a$ inhibitors.

43. - Samama MM, Contant G, Spiro TE, et al. Evaluation of the prothrombin time for measuring rivaroxaban plasma concentrations using calibrators and controls: results of a multicenter field trial. Clin Appl Thromb Hemost. 2012;18:150-8. This study is one of the first to demonstrate a potential ability to monitor direct FXa therapy, rivaroxaban, using the readily available PT. Most previous papers describe the qualitative ability of this test, but now with the use of the calibrators and controls used in this study, a more quantitative assessment can be achieved.

44. Gerotziafas GT, Hatmi M, Samama MM, Elalamy I. Optimisation of the assay for the measurement of clotting factor activity in the presence of rivaroxaban. Thromb Res. 2012;129:101-3.

45. Funatsu T, Yamashita A, Kaku S, et al. Plasma factor Xa inhibition can predict antithrombotic effects of oral direct factor Xa inhibitors in rabbit atherothrombosis models. Thromb Haemost. 2012;108:896-902.

46. Wong PC, Crain EJ, Xin B, et al. Apixaban, an oral, direct and highly selective factor Xa inhibitor: in vivo, antithrombotic and antihemostatic studies. J Thromb Haemost. 2008;6:820-9.

47. Tobu M, Iqbal O, Hoppensteadt D, et al. Anit-Xa and anti-IIa drugs alter international normalized ratio measurement: potential problems in monitoring of oral anticoagulants. Clin Appl Thromb Hemost. 2004;10:301-9.
48. Fenyvesi T, Jörg I, Harenberg J. Monitoring of anticoagulant effect of direct thrombin inhibitors. Semin Thromb Hemost. 2002;28:361-8.

49. Love JE, Ferrell C, Chandler WL. Monitoring direct thrombin inhibitors with a plasma diluted thrombin time. Thromb Haemost. 2007;98:234-42.

50. Avecilla ST, Ferrell C, Chandler WL, Reyes M. Plasma-diluted thrombin time to measure dabigatran concentrations during dabigatran etexilate therapy. Am J Clin Pathol. 2012;137:572-4.

51. Freyburger G, Macouillard G, Labrouche S, Sztark F. Coagulation parameters in patients receiving dabigitran etexilate or rivaroxaban: two observational studies in patients undergoing total hip or total knee replacement. Thromb Res. 2011;127:457-65.

52. Stangier J, Feuring M. Using the HEMOCLOT direct thrombin inhibitor assay to determine plasma concentrations of dabigatran. Blood Coagul Fibrinolysis. 2012;23:138-43.

53. Nowak G. The ecarin clotting time, a universal method to quantify direct thrombin inhibitors. Pathophysiol Haemost Thromb. 2003;33:173-83.

54. Lange U, Nowak G, Bucha E. Ecarin chromogenic assay-a new method for quantitative determination of direct thrombin inhibitors like hirudin. Pathophysiol Haemost Thromb. 2004;33:184-91.

55. Walenga JM, Hoppensteadt DA. Monitoring the new antithrombotic drugs. Semin Thromb Hemost. 2004;30:683-95.

56. Amis LM, Alberio L, Angelillo-Scherrer A, et al. Rivaroxaban: quantification by anti-FXa assay and influence on coagulation tests. A study in 9 Swiss laboratories. Thromb Res. 2012;129:492-8.

57. Samama MM, Amiral J, Guinet C, et al. An optimized, rapid chromogenic assay specific for measuring direct factor Xa inhibitors (rivaroxaban) in plasma. Thromb Haemost. 2010;104:1078-9.

58. - Samama MM, Contant G, Spiro TE, et al. Evaluation of the antifactor Xa chromogenic assay for the measurement of rivaroxaban plasma concentrations using calibrators and controls. Thromb Haemost 2012;107:379-87. This study evaluated several assays for measuring rivaroxaban. There was a dose-dependant relationship between anti-FXa activity and rivaroxaban concentration with the chromogenic assay, suggesting it may be useful for monitoring.

59. Harenberg J, Giese C, Marx S, Krämer R. Determination of dabigatran in human plasma samples. Semin Thromb Hemost. 2012;38:16-22. 